\title{
High-Level Synthesis through Transforming VHDL Models
}

\author{
Anatoly Prihozhy \\ Belarusian State Polytechnic \\ aprihozhy@bspa.unibel.by
}

\begin{abstract}
In this paper a method of transforming a behavioral VHDL-model to a functionally equivalent model with one basic block is proposed. High-level synthesis techniques including scheduling, allocation, and binding are modified for the model. These reduce the number of control steps, FSM states, state transitions, functional and storage units in an RTL-structure.
\end{abstract}

\section{Introduction}

Usually, the designer starts by describing the required behavior and specifying the optimization goal and design constraints. Capturing this input, a high-level synthesis tool generates a structure at the register transfer level (RTL) [1-5]. If the designer is not satisfied with the synthesis result, she/he can modify the behavioral description, optimization criterion, or design constraints and try again. Nowadays the path-based high-level synthesis method is widely and successfully used in efficient synthesis tools [1-5,10,11]. It constitutes a theoretical basis for performing data-control flow graph (DCFG) analysis, scheduling, allocation, and binding for the whole behavior. At the same time, the method has the drawbacks as follows:

- The number of paths on the CFG can increase exponentially depending on the number of nodes (up to millions of paths are possible for real designs [10])

- Reordering of statements in a path is not allowed

- During scheduling, allocation and binding very complex combinatorial tasks have to execute for each path and the obtained results have to be combined.

In this paper we prove that any behavioral description can be equivalently transformed to a one basic block model (OBBM) allowing more powerful synthesis results than the traditional approaches allow. Section 2 presents the idea of transformational synthesis. In sections 3 and 4 transformation rules and techniques for inferring OBBM are described. Scheduling, allocation, and binding techniques for OBBM are presented in sections 5 and 6 . Section 7 gives some results.

\section{Transformation-based synthesis}

Starting from a behavioral VHDL-description, we generate a number of equivalent descriptions [6-9] by applying transformations (Fig.1). The technique extends the set of design alternatives and supports an efficient design space exploration (Fig.2). In this paper we propose a method of transforming a behavioral VHDL-model to a functionally equivalent model constructed of one basic block. The existing scheduling, allocation and binding techniques can't be directly applied to the model. We modify the techniques by means of using several relations on the sets of variables and statements in order to improve parameters of the generated RTL-structure.

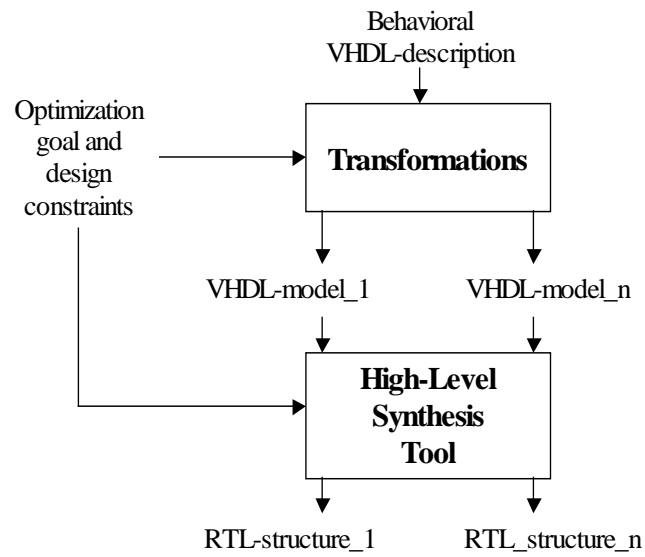

Figure 1. Synthesis through transformation

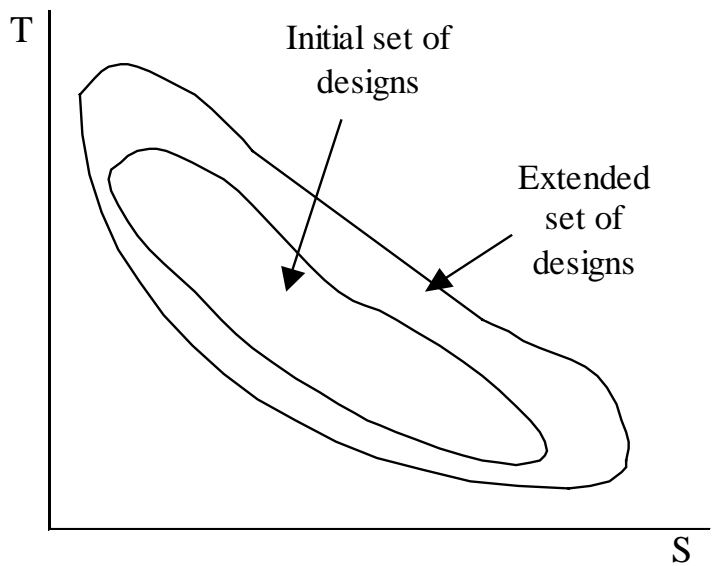

Figure 2. Design space exploration 


\section{Transformation of original model}

We transform a behavioral sequential synchronous VHDL model with flexible or specified cycle behavior in order to obtain a VHDL-model that is more suitable for efficient high-level synthesis. Several types of useful transformations are used in the existing HLS tools [1-11]. We propose deeper transformations leading to significant reorganization of the source VHDL-model and its CFG: splitting statements, inserting statements, extracting computations, attaching statements, eliminating loops, eliminating exit- and next-statements, and others. The algebraic methods of transforming Boolean expressions will be intensively used as well. We will avoid multiple assignments to the same variable. Through the paper the following notations will be used: $V, V 1, \ldots, C, C 1, \ldots$ are Boolean variables and expressions, $Q, Q 1, \ldots$ are sequential statements, $S, S 1, \ldots$ are sequences of statements, and $L, L 1, \ldots$ are labels of loops.

\subsection{Wait-statements}

In the sequential synchronous behavioral model a sequence of statements may describe a specified cycle behavior or a flexible cycle behavior [1]. The VHDL-statement

wait until C and Clock'Event and Clock='1';

is used to control the specified cycle behavior, where Clock is the signal triggering the state transition. The fragment is equivalent to the loopstatement:

loop wait until Clock'Event and Clock='1'; exit when $C$; end loop;

\subsection{Loop-statements}

Our transformation rules are significantly based on using loop-statements without an iteration scheme: L: loop $S$ end loop L;. The VHDL loop-statements while and for with ascending and descending ranges as follows:

while $C$ loop $S$ end loop;

for $I$ in $L$ to $R$ loop $S$ end loop;

for $I$ in $L$ downto $R$ loop $S$ end loop

may be replaced with

loop $\mathrm{V}:=\operatorname{not} \mathrm{C}$; exit when $\mathrm{V}$; $\mathrm{S}$ end loop;

$\mathrm{I}:=\mathrm{L}$; loop $\mathrm{V}:=\mathrm{I}>\mathrm{R}$; exit when $\mathrm{V}$; $\mathrm{S} \mathrm{I}:=\mathrm{I}+1$; end loop;

$\mathrm{I}:=\mathrm{L}$; loop $\mathrm{V}:=\mathrm{I}<\mathrm{R}$; exit when $\mathrm{V}$; $\mathrm{S} \mathrm{I}:=\mathrm{I}-1$; end loop;

Very often a loop-statement can be under an ifthen-statement as in the following fragment:

if $C$ then loop $S$ end loop; end if;

Inserting the if-statement into the loop-statement yields:

$\mathrm{V}:=$ true; loop $\mathrm{V} 1:=\mathrm{V}$ and $\operatorname{not} \mathrm{C} ; \mathrm{V}:=$ false; exit when $V 1 ; S$ end loop;.

\subsection{Exit-statements}

An exit-statement works together with a loop: $L$ : loop $S 1$ exit $L$ when $C$; $S 2$ end loop;. If an exitstatement is under an if-statement:

if $\mathrm{C} 1$ then exit $\mathrm{L}$ when $\mathrm{C} 2$; end if;

then the two statements may be merged as

$\mathrm{V}:=\mathrm{C} 1$ and $\mathrm{C} 2$; exit $\mathrm{L}$ when $\mathrm{V}$;

If an exit-statement exit $L$ when $C ; S$ is followed by a sequence $S$ of statements that does not update the value of $C$, then the two statements may be reordered as:

if not $C$ then $S$ end if; exit $L$ when $C$;

Two exit-statements with the same loop-label:

exit $L$ when $C 1$; exit $L$ when $C 2$;

may be merged as:

$\mathrm{V}:=\mathrm{C} 1$ or $\mathrm{C} 2$; exit $\mathrm{L}$ when $\mathrm{V}$;

\subsection{Next-statements}

All the next-statements are eliminated from the VHDL-behavioral description. If a next-statement is under an if-statement:

if $C 1$ then next $L$ when $C 2$; end if;

then the two statements may be merged as

$\mathrm{V}:=\mathrm{C} 1$ and $\mathrm{C} 2$; next $\mathrm{L}$ when $\mathrm{V}$;

The most general situation with a next-statement and two nested loops can be represented as:

L1: loop S1 L2: loop S2 next L1 when C; S3 end loop L2; S4 end loop L1;

Replacing the next-statement with exit- and ifstatements yields:

V:=false; L1: loop S1 L2: loop S2 V:=C;

exit $L 2$ when $V$; $S 3$ end loop $L 2$;

if not $\mathrm{V}$ then $\mathrm{S} 4$ end if; end loop $\mathrm{L} 1$;

The same transformation can be performed on an arbitrary number of nested loops. It can imply introducing a label for an unlabeled loop.

\subsection{Return-statements}

The return-statements are used to exit from VHDL functions and procedures. We eliminate the statements from models and replace them with variable assignment, exit- and if-then-statements. 


\subsection{If-statements}

An if-statement in VHDL selects no more than one sequence of statements and is represented in the following form:

if $\mathrm{C} 1$ then $\mathrm{S} 1$ elsif $\mathrm{C} 2$ then $\mathrm{S} 2 \bullet \bullet \bullet$ elsif $C n-1$ then $S n-1$ else $S n$ end if;

The if-statement may be split to the following sequence of variable assignment and if-thenstatements:

$\mathrm{V} 1:=\mathrm{C} 1 ; \mathrm{V} 2:=\operatorname{not} \mathrm{C} 1$ and $\mathrm{C} 2 ; \bullet \bullet \bullet$

$\mathrm{Vn}-1:=\operatorname{not} \mathrm{C} 1$ and not $\mathrm{C} 2$ and $\ldots$ and $\mathrm{Cn}-1$;

$\mathrm{Vn}:=\operatorname{not} \mathrm{C} 1$ and not $\mathrm{C} 2$ and $\ldots$ and not $\mathrm{Cn}-1$; if $\mathrm{V} 1$ then $\mathrm{S} 1$ end if; if $\mathrm{V} 2$ then $\mathrm{S} 2$ end if; $\bullet \bullet \bullet$ if $\mathrm{Vn}-1$ then $\mathrm{Sn}-1$ end if; if $\mathrm{Vn}$ then $\mathrm{Sn}$ end if;

The if-then-statement if $V$ then $Q 1 ; \ldots Q k$; end if; containing a sequence of other statements may be split to a sequence of if-then-statements each of them with one statement inside:

if $\mathrm{V}$ then $\mathrm{Q} 1$; end if; $\ldots$ if $\mathrm{V}$ then $\mathrm{Qk}$; end if;

This transformation is eligible if $V$ is not reassigned in $Q 1, \ldots, Q k-1$. If one if-statement is inside of another if-statement then the described splitting procedure can yield if $V 1$ then if $V 2$ then $Q$; end if; end if; that is reduced to

$\mathrm{V}:=\mathrm{V} 1$ and $\mathrm{V} 2$; if $\mathrm{V}$ then $\mathrm{Q}$; end if;

In special case if $V 1$ then $V 2:=E$; end if; the ifthen-statement may be replaced with a variable assignment statement:

$\mathrm{V} 2:=(\mathrm{V} 1$ and $\mathrm{E})$ or $($ not V1 and V2);

Very often two VHDL-fragments as follows will appear during transformations:

$\mathrm{V} 2:=$ false; if $\mathrm{V} 1$ then $\mathrm{V} 2:=\mathrm{E}$; end if;

$\mathrm{V} 2:=$ true; if $\mathrm{V} 1$ then $\mathrm{V} 2:=\mathrm{E}$; end if

These may be replaced with:

$\mathrm{V} 2:=\mathrm{V} 1$ and $\mathrm{E}$;

$\mathrm{V} 2:=\mathrm{E}$ or not $\mathrm{V} 1$.

If $E$ implies $V 1$ in (3.20), that is $E \rightarrow V 1$ holds for all the values of primary variables, and the previous value of $V 2$ is not determined, then (3.20) is reduced to $V 2:=E$;. Here ' $\rightarrow$ ' is the logical implication operation. Similarly, if the implication $V 2 \rightarrow V 1$ holds then (3.19) is reduced to:

if $\mathrm{V} 2$ then $\mathrm{Q}$; end if;

\subsection{Case-statements}

In VHDL the case statement has the following form: case $\mathrm{E}$ is when $\mathrm{H} 11|\ldots| \mathrm{H} 1 \mathrm{k}_{1} \Rightarrow \mathrm{S} 1 \bullet \bullet \bullet$

when $\mathrm{Hr} 1|\ldots| \mathrm{Hrk}_{\mathrm{r}} \Rightarrow>\mathrm{Sr}$ when others $\Rightarrow \mathrm{Sr}+1$ end case;

where $E$ is an expression of a discrete type and $H i j$ is a choice defining a value or a range of values. The case-statement is equivalently transformed to the following if-statement:

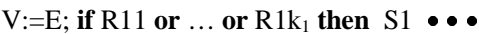

elsif $\mathrm{Rr}$ or ... or $\mathrm{Rrk}_{\mathrm{r}}$ then $\mathrm{Sr}$ else $\mathrm{Sr}+1$ end if;

where $V$ is a variable of the type associated with the expression $E$ and $R i j$ is a relational operator (expression) associated with the choice Hij. The ifstatement is split as it was described in section 3.6.

\subsection{Variable and signal assignments}

A variable assignment statement with an aggregate in the left part is split into a sequence of assignment statements with a variable in the left part. If an assignment statement contains an expression $E$ in the right part constructed of more than one operator, it is split implying the addition of variables of appropriate types. A signal assignment statement using operators in the right part is replaced with a signal assignment statement without operators by means of introducing an additional variable and variable assignment statement.

\subsection{Procedure and function calls}

All the transformation rules that are considered in the paper may be applied to the processes and subprograms. In particular, we can represent the subprogram body as one basic block. Two approaches to processing the subprograms during high-level synthesis are possible: performing highlevel synthesis separately for each subprogram and merging the process and subprograms before synthesis. A drawback of the first approach is that it is not easy to optimize the design cost. A drawback of the second approach is that it is desirable to avoid multiple attaching the subprogram body to the process body because the VHDL-code can extend significantly when a lot of calls of the same subprogram exist. In order to unify the merging algorithm, all the functions are replaced with procedures and assignment statements $U:=F(P)$; are replaced with procedure calls $F(P, U)$ where $U$ is a variable, $F$ is a function name, and $P$ is a list of actual parameters.

\section{VHDL-model with one basic block}

The number of paths on CFG significantly depends on the number of basic blocks. In this section we prove that any VHDL-process may be represented as one basic block in the statement part: 
P: process Declars

begin Basic_Block end process $P$;

where Declars are local declarations and Basic_Block is a sequence of wait, variable and signal assignment statements either covered or not covered with if-then-statements. An if-thenstatement describes a condition of operation execution and variable/signal assignment. The process parenthesis "begin" and "end" describe an infinite loop. The proposed transformation technique consists of the key steps as follows: inserting statements located after a loop into the loop, inserting statements located before a loop into the loop, merging neighbor nested loops, eliminating loop- and exit-statements, and eliminating subprogram calls.

\subsection{Inserting statements into loop}

We assume that all the loop-statements are labeled and all the exit-statements refer a loop-label. While inserting statements located after a loop, the number of exit-statements in the loop has to be taken into account as in the following VHDLfragment:

L: loop S1 exit L when V1; S2 exit $L$ when V2; S3 end loop L; S4

where $S 4$ is a sequence of statements that do not update the value of variables $V 1$ and $V 2$ (otherwise we can use additional variables). Inserting $S 4$ into the loop yields:

$\mathrm{L}$ : loop $\mathrm{S} 1$ if $\mathrm{V} 1$ then $\mathrm{S} 4$ end if; exit $\mathrm{L}$ when $\mathrm{V} 1$; $\mathrm{S} 2$ if $\mathrm{V} 2$ then $\mathrm{S} 4$ end if; exit $\mathrm{L}$ when $\mathrm{V} 2 ; \mathrm{S} 3$ end loop $\mathrm{L}$;

The drawback is that two copies of $S 4$ appear in the loop-body. In order to have one copy we begin with reordering and merging the exit-statements:

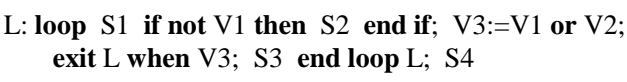

and then the sequence $S 4$ is inserted into the loop:

L: loop $\mathrm{S} 1$ if not $\mathrm{V} 1$ then $\mathrm{S} 2$ end if; $\mathrm{V} 3:=\mathrm{V} 1$ or $\mathrm{V} 2$; (4.5) if $\mathrm{V} 3$ then $\mathrm{S} 4$ end if; exit $\mathrm{L}$ when $\mathrm{V} 3$; $\mathrm{S} 3$ end loop $\mathrm{L}$;

The technique is eligible for an arbitrary number of exit-statements in one loop. When statements located before a loop are inserted into the loop, the exit-statements are not used, so we consider the VHDL-fragment as:

S1 L: loop S2 end loop L;

that is functionally equivalent to:

$\mathrm{V}:=$ true; L: loop

if $V$ then $S 1$ end if; $V:=$ false; $S 2$ end $\operatorname{loop} L$;

If the loop-statement is under an if-then-statement:

$S 1$ if $C$ then $L$ : loop S2 end loop L; end if; then the inserting yields:

$\mathrm{V}:=$ true; $\mathrm{L}$ : loop if $\mathrm{V}$ then $\mathrm{S} 1$ end if;

$\mathrm{V} 1:=\mathrm{V}$ and not $\mathrm{C} ; \mathrm{V}:=$ false; exit when $\mathrm{V} 1 ; \mathrm{S} 2$ end loop $\mathrm{L}$;

Inserting of statements located after a loop is cheaper than inserting of statements located before the loop.

\subsection{Generating nested loops}

Usually a VHDL-process or subprogram contains a hierarchy of loops. In the hierarchy pairs of loops exist that either execute sequentially or one loop is in the body of other loop. For the both cases we prove that an equivalent system of nested loops may be constructed. First, we transform the VHDL-fragment:

L1: loop $\mathrm{S} 1$ if $\mathrm{C} 1$ then $\mathrm{L} 2$ : loop $\mathrm{S} 2$ exit $\mathrm{L} 2$ when $\mathrm{C} 2 ;(4.10)$ $\mathrm{S} 3$ end loop L2; end if; S4 end loop L1;

where the loop $L 2$ is in the body of the loop $L 1$ and is covered by an if-then-statement. After inserting the sequences $S 1$ and $S 4$ into the loop $L 2$ and attaching the exit-statements we have:

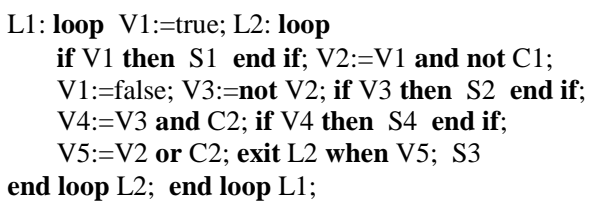

Second, we transform the sequence of two loops:

L1: loop S1 exit L1 when C1; S2 end loop L1;

S3 L2: loop S4 exit L2 when C2; S5 end loop L2;

After inserting the loop L2 into the loop L1 and inserting the statements located before $L 2$ into the loop we have:

L1: loop V1:=true; L2: loop

if $\mathrm{V} 1$ then $S 1$ end if: $V 2:=V 1$ and $C 1$

if $\mathrm{V} 2$ then $\mathrm{S} 3$ end if; $\mathrm{V} 3:=\mathrm{V} 1$ and $\operatorname{not} \mathrm{C} 1$;

$\mathrm{V} 1:=$ false; exit L2 when V3; S4 exit L2 when $\mathrm{C} 2$; S5 end loop L2; exit L1 when $\mathrm{C} 1$; S2 end loop L1;

Merging two exit-statements referring label $L 2$ and insert-ing the statements located after $L 2$ into the loop yields:

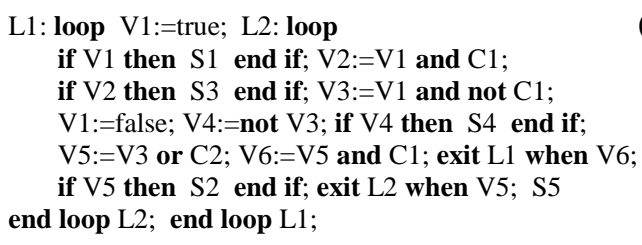

Consecutively applying the described transformations to pairs of hierarchical and sequential loops, we can transform any VHDLprocess with an arbitrary number and structure of loops to a process with nested loops. 


\subsection{Eliminating loop- and exit-statements}

Two nested loops:

L1: loop V1:=true; L2: loop S1 exit L2 when C1;

S2 end loop L2; end loop L1;

may be replaced with one loop:

$\mathrm{L} 1$ : loop $\mathrm{S} 1$ if $\mathrm{C} 1$ then $\mathrm{V} 1:=$ true; end if;

$\mathrm{V} 2:=\operatorname{not} \mathrm{C} 1$; if $\mathrm{V} 2$ then $\mathrm{S} 2$ end if; end loop $\mathrm{L} 1$;

if the variable $V 1$ used during inserting statements takes initial value true. The exit-statement is replaced with a conditional (C1) assignment of value true to the control variable $V 1$. The sequence $S 2$ also executes conditionally, but with the condition not C1. Applying the rule to nested loops (4.11) inferred from two hierarchical loops (4.10) and using rules (3.20) to (3.23), we obtain one loop as follows:

$\mathrm{L} 1:$ loop if $\mathrm{V} 1$ then $\mathrm{S} 1$ end if; $\mathrm{V} 2:=\mathrm{V} 1$ and $\operatorname{not} \mathrm{C} 1$;

$\mathrm{V} 3:=$ not $\mathrm{V} 2$; if $\mathrm{V} 3$ then $\mathrm{S} 2$ end if;

$\mathrm{V} 4:=\mathrm{V} 3$ and $\mathrm{C} 2$; if $\mathrm{V} 4$ then $\mathrm{S} 4$ end if;

$\mathrm{V} 1:=\mathrm{V} 2$ or $\mathrm{C} 2$; $\mathrm{V} 5:=$ not $\mathrm{V} 1$; if $\mathrm{V} 5$ then $\mathrm{S} 3$ end if; end loop L1;

Similarly, nested loops (4.14) inferred from sequential loops (4.12) are transformed to one loop:

$\mathrm{L} 1$ : loop if $\mathrm{V} 1$ then $\mathrm{S} 1$ end if; $\mathrm{V} 2:=\mathrm{V} 1$ and $\mathrm{C} 1$; if $\mathrm{V} 2$ then $\mathrm{S} 3$ end if; $\mathrm{V} 7:=\operatorname{not} \mathrm{C} 1$; $\mathrm{V} 3:=\mathrm{V} 1$ and $\mathrm{V} 7$; $\mathrm{V} 4:=$ not $\mathrm{V} 3$; if $\mathrm{V} 4$ then $\mathrm{S} 4$ end if; $\mathrm{V} 1:=\mathrm{V} 3$ or $\mathrm{C} 2 ; \mathrm{V} 6:=\mathrm{V} 1$ and $\mathrm{C} 1$; exit $\mathrm{L} 1$ when $\mathrm{V} 6$; if $\mathrm{V} 1$ then $\mathrm{S} 2$ end if; $\mathrm{V} 5:=$ not $\mathrm{V} 1$; if $\mathrm{V} 5$ then $\mathrm{S} 5$ end if; end loop L1;

Given a process with $k$ nested loops the loops may be eliminated step by step. Finally, a process with one basic block is inferred. The process statement part is a loop that is suspended and resumed by events on the Clock signal.

\subsection{Eliminating subprogram calls}

We assume that a subprogram is called for more than once in a process (another subprogram) body. Let the bodies of subprogram and process be represented as one basic block. If each subprogram call is replaced with the subprogram body of significant size, the VHDL code can extend significantly. Our goal is to construct the process body in such a way to attach one copy of the subprogram body. The goal is achieved through using additional control Boolean variables and ifstatements. We illustrate our approach with the following abstract VHDL-like fragment:

process $\bullet \bullet \bullet$

procedure $\mathrm{F}(\mathrm{Pi}, \mathrm{Po})$ is Declars begin $\mathrm{B}$ end $\mathrm{F}$; begin $\mathrm{S} 1 \mathrm{~F}(\mathrm{P} 1 \mathrm{i}, \mathrm{P} 1 \mathrm{o})$; $\mathrm{S} 2 \mathrm{~F}(\mathrm{P} 2 \mathrm{i}, \mathrm{P} 2 \mathrm{o})$; $\mathrm{S} 3$ end process;

where $F$ is a procedure name, $P i$ and $P o$ are descriptions of input and output formal parameters, Declars is a list of declarative items, $B$ is a procedure body represented as one basic block, and $P 1 i, P 1 o, P 2 i$, and $P 2 o$ are input and output actual parameters of first and second procedure calls. After inserting the declarations $P i, P o$, and Declars into the process declarative part, inserting the procedure body $B$ into the process statement part, and reorganizing the statement part, the fragment is as follows:

process $\bullet \bullet P$ Pi, Po, and Declars modified

variable $\mathrm{V} 1, \ldots, \mathrm{V} 7$ : Boolean; begin

$\mathrm{V} 3:=\mathrm{V} 1$ or $\mathrm{V} 2$; if $\mathrm{V} 3$ then $\mathrm{B}$ end if;

$\mathrm{V} 4:=$ not $\mathrm{V} 3$; if $\mathrm{V} 4$ then $\mathrm{S} 1$ end if;

if $\mathrm{V} 4$ then $\mathrm{Pi} ':=\mathrm{P} 1 \mathrm{i}$; end if; $\mathrm{V} 5:=\mathrm{V} 1$ and not $\mathrm{V} 2$;

if $\mathrm{V} 5$ then $\mathrm{P} 1 \mathrm{o}:=\mathrm{Po}$; end if; if $\mathrm{V} 5$ then $\mathrm{S} 2$ end if;

if $\mathrm{V} 5$ then $\mathrm{Pi} \mathrm{i}^{\prime}:=\mathrm{P} 2 \mathrm{i}$; end if; $\mathrm{V} 6:=$ not $\mathrm{V} 1$ and $\mathrm{V} 2$;

if $\mathrm{V} 6$ then $\mathrm{P} 2 \mathrm{o}:=\mathrm{Po}$; end if; if $\mathrm{V} 6$ then $\mathrm{S} 3$ end if;

$\mathrm{V} 7:=\mathrm{V} 1$ xor $\mathrm{V} 2 ; \mathrm{V} 2:=\mathrm{V} 1 ; \mathrm{V} 1:=$ not $\mathrm{V} 7$;

end process;

where $P{ }^{\prime}$ ' and $P o$ ' are local variables representing the input and output formal parameters of procedure. In the process, all the variables that are used in $B$ have to be unique. Increase in the number of procedure calls requires additional control variables, although the transformation method remains the same.

\subsection{An example}

Now we demonstrate the proposed transformation technique on a simple VHDL behavioral description. An original GCD algorithm is presented in Fig.3. First, we split all the control structures and remove the iteration scheme from the loop (Fig.4). Three additional Boolean variables $C l, C 2$, and $C 3$ are introduced. Then, according to (4.5) and (4.7) we insert the statements that precede and succeed the loop into the loop (Fig.5). An additional variable $C O$ is used. After that the loop is eliminated, the exit-statement is replaced with an if-statement, and the variable $\mathrm{CO}$ takes the initial value true (Fig.6). Finally, two statements that assign a value to variable $C O$ are merged and the resulting variable assignment statement proves that $C O=C l$. The variable $C l$ is removed and replaced with $C O$ (Fig.7). The waveforms of input and output signals are the same for all the VHDL-models (Fig.8). Any behavioral VHDL-description can be transformed in the similar way.

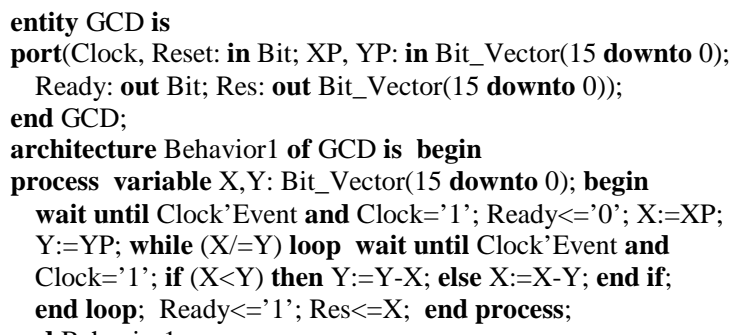

Figure 3. Original VHDL-model of GCD 
architecture Behavior2 of GCD is begin

process variable $\mathrm{X}, \mathrm{Y}$ : $\mathrm{Bit}$ _Vector( 15 downto 0$)$;

variable $\mathrm{C} 1, \mathrm{C} 2, \mathrm{C} 3$ : Boolean; begin

wait until Clock'Event and Clock='1'; Ready<='0' X:=XP;

$\mathrm{Y}:=\mathrm{YP}$; loop $\mathrm{C} 1:=\mathrm{X}=\mathrm{Y}$; exit when $\mathrm{C} 1$;

wait until Clock'Event and Clock $={ }^{\prime} 1^{\prime} ; \mathrm{C} 2:=\mathrm{X}<\mathrm{Y} ; \mathrm{C} 3:=\operatorname{not} \mathrm{C} 2$;

if $\mathrm{C} 2$ then $\mathrm{Y}:=\mathrm{Y}-\mathrm{X}$; end if; if $\mathrm{C} 3$ then $\mathrm{X}:=\mathrm{X}-\mathrm{Y}$; end if;

end loop; Ready<='1'; Res $<=X$; end process;

end Behavior2;

Figure 4. Transformed VHDL-model (step 1)

architecture Behavior3 of GCD is begin process

variable $X, Y$ : Bit_Vector(15 downto 0$)$;

variable $\mathrm{C} 0, \mathrm{C} 1, \mathrm{C} 2, \mathrm{C} 3$ : Boolean; begin $\mathrm{C} 0$ :=true; loop if $\mathrm{C} 0$ then wait until Clock'Event and Clock=' 1 '; end if; if $\mathrm{C} 0$ then Ready $\angle=' 0$ '; end if; if $\mathrm{C} 0$ then $\mathrm{X}:=\mathrm{XP}$; end if if $\mathrm{C} 0$ then $\mathrm{Y}:=\mathrm{YP}$; end if; $\mathrm{C} 0:=$ false; $\mathrm{C} 1:=\mathrm{X}=\mathrm{Y}$; if $\mathrm{C} 1$ then $\mathrm{Ready}<=$ ' 1 '; end if; if $\mathrm{C} 1$ then $\operatorname{Res}<=\mathrm{X}$; end if exit when $\mathrm{C} 1$; wait until Clock'Event and Clock='1'; $\mathrm{C} 2:=\mathrm{X}<\mathrm{Y} ; \mathrm{C} 3:=$ not $\mathrm{C} 2$; if $\mathrm{C} 2$ then $\mathrm{Y}:=\mathrm{Y}-\mathrm{X}$; end if; if $\mathrm{C} 3$ then $\mathrm{X}:=\mathrm{X}-\mathrm{Y}$; end if; end loop; end process; end Behavior3;

Figure 5. Transformed VHDL-model (step 2)

architecture Behavior4 of GCD is begin process

variable $X, Y$ : Bit Vector(15 downto 0 );

variable $\mathrm{C} 0, \mathrm{C} 1, \mathrm{C} 2, \mathrm{C} 3$ : Boolean:=true; begin

if $\mathrm{C} 0$ then wait until Clock'Event and Clock='1'; end if if $\mathrm{C} 0$ then Ready $\angle={ }^{\prime}$ '; end if; if $\mathrm{C} 0$ then $\mathrm{X}:=\mathrm{XP}$; end if; if $\mathrm{C} 0$ then $\mathrm{Y}:=\mathrm{YP}$; end if; $\mathrm{C} 0:=$ false; $\mathrm{C} 1:=\mathrm{X}=\mathrm{Y}$;

if $\mathrm{C} 1$ then $\operatorname{Ready}<=$ ' 1 '; end if; if $\mathrm{C} 1$ then $\operatorname{Res}<=\mathrm{X}$; end if; if $\mathrm{C} 1$ then $\mathrm{C} 0:=$ true; end if; if not $\mathrm{C} 1$ then wait until

Clock'Event and Clock='1'; end if; $\mathrm{C} 2:=\mathrm{X}<\mathrm{Y} ; \mathrm{C} 3:=\mathrm{X}>\mathrm{Y}$;

if $\mathrm{C} 2$ then $\mathrm{Y}:=\mathrm{Y}-\mathrm{X}$; end if; if $\mathrm{C} 3$ then $\mathrm{X}:=\mathrm{X}-\mathrm{Y}$; end if;

end process; end Behavior4;

Figure 6. Transformed VHDL-model (step 3)

architecture Behavior5 of GCD is begin process

variable $\mathrm{X}, \mathrm{Y}$ : Bit Vector(15 downto 0$)$;

variable $\mathrm{C} 0, \mathrm{C} 2, \mathrm{C} 3$ : Boolean:=true; begin

if $\mathrm{C} 0$ then wait until Clock'Event and Clock=' 1 '; end if if $\mathrm{C} 0$ then Ready $\angle={ }^{\prime}$ '; end if; if $\mathrm{C} 0$ then $\mathrm{X}:=\mathrm{XP}$; end if; if $\mathrm{C} 0$ then $\mathrm{Y}:=\mathrm{YP}$; end if; $\mathrm{C} 0:=\mathrm{X}=\mathrm{Y}$;

if $\mathrm{C} 0$ then Ready<=' 1 '; end if; if $\mathrm{C} 0$ then $\operatorname{Res}<=\mathrm{X}$; end if if not $\mathrm{C} 0$ then wait until Clock'Event and Clock='1'; end

if; $\mathrm{C} 2:=\mathrm{X}<\mathrm{Y}$; $\mathrm{C} 3:=\mathrm{X}>\mathrm{Y}$; if $\mathrm{C} 2$ then $\mathrm{Y}:=\mathrm{Y}-\mathrm{X}$; end if;

if $C 3$ then $X:=X-Y$; end if; end process;

end Behavior5;

Figure 7. Transformed VHDL-model (step 4)

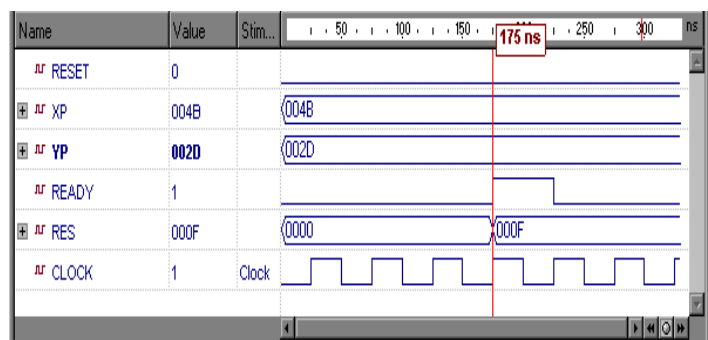

Figure 8. Signal waveforms for GCD

\section{Scheduling for OBBM}

Scheduling aims at introducing control steps and FSM states, distributing statements on the steps, and either minimizing the number of steps or minimizing the design cost. The scheduling results in generating a high-level state machine HLSM. The known scheduling techniques that process a traditional basic block are [1-5]: as soon as possible ASAP, as late as possible ALAP, list scheduling, integer linear programming formulation ILPF, freedom-based scheduling, force-directed scheduling, dynamic loop scheduling, scheduling for pipelines and other techniques. The path-based scheduling technique processes the whole CFG consisting of several basic blocks.

\subsection{Scheduling methodology}

In a traditional basic block all statements execute unconditionally. In the OBBM a statement may execute conditionally or unconditionally. Our scheduling methodology includes: analyzing data dependencies, generating orthogonal, implication, and equivalence relations on the set of control signals and variables, generating a set of pairs of orthogonal statements, analyzing operator compatibility and operator proximity, generating a statement precedence relation, performing a modified scheduling technique (ILPF, list scheduling, ASAP, and ALAP). The generated schedule is optimized due to orthogonal operations are mutually exclusive and may execute on the same functional unit concurrently without additional resources. As a result, the statements are reordered, grouped, and an optimized HLSM is generated.

\subsection{Relations on the set of variables}

Signals and variables (mostly variables) of Boolean type control the execution of if-thenstatements. Relations on the set of control variables define in which manner the operators and assignments that are under an if-then-statement execute. Two control variables $V 1$ and $V 2$ are:

- Orthogonal (\#) if the variables take never value true simultaneously

- Variable V1 implies variable $V 2(\rightarrow)$ if $V 2$ takes never value false when $V 1$ equals true

- Variable $V 2$ implies variable $V 1(\leftarrow)$ if $V 1$ takes never value false when $V 2$ equals true

- Equivalent $(\leftrightarrow)$ if the variables can't take different values simultaneously

- Independent (-) if neither of the previous cases takes place.

There are two main sources for inferring the relations. First, the VHDL relational operators are analyzed. Given two assignment statements $V 1:=X$ $R 1 Y$; and $V 2:=X R 2 Y$; with the same operands where $R 1, R 2 \in\{=,=,<,<=>,>=\}$ are relational operators, the relations \#, $\rightarrow, \leftarrow$, and $\leftrightarrow$ between $V 1$ and $V 2$ are inferred through using Table 1. The similar table is used for a pair of statements $V 1:=X$ $R 1 L 1$; and $V 2:=X R 2 L 2$; where $L 1$ and $L 2$ are 
Inferring relations between $V 1$ and $V 2$

Table 1

\begin{tabular}{|c|c|c|c|}
\hline N & $\begin{array}{c}\text { Operator } \\
R 1\end{array}$ & $\begin{array}{c}\text { Operator } \\
R 2\end{array}$ & $\begin{array}{c}\text { Relation between } \\
V 1 \text { and } V 2\end{array}$ \\
\hline 1 & $=$ & $=$ & $\leftrightarrow$ \\
\hline 2 & $=$ & $/=,<,>$ & $\#$ \\
\hline 3 & $=$ & $<=,>=$ & $\#$ \\
\hline 4 & $/=$ & $=$ & $\leftrightarrow$ \\
\hline 5 & $/=$ & $/=$ & $\leftarrow$ \\
\hline 6 & $/=$ & $<,>$ & $\#$ \\
\hline 7 & $<$ & $=,>,>=$ & $\rightarrow$ \\
\hline 8 & $<$ & $/=,<=$ & $\leftrightarrow$ \\
\hline 9 & $<$ & $<$ & $\leftarrow$ \\
\hline 10 & $<=$ & $=,<$ & $\leftrightarrow$ \\
\hline 11 & $<=$ & $<=$ & $\#$ \\
\hline 12 & $<=$ & $>$ & $\#$ \\
\hline 13 & $>$ & $=,<,<=$ & $\rightarrow$ \\
\hline 14 & $>$ & $/=,>=$ & $\leftrightarrow$ \\
\hline 15 & $>$ & $>$ & $\leftarrow$ \\
\hline 16 & $>=$ & $=,>$ & $\leftrightarrow$ \\
\hline 17 & $>=$ & $<$ & $\leftrightarrow$ \\
\hline 18 & $>=$ & $>=$ & \\
\hline & & & $\leftrightarrow$ \\
\hline
\end{tabular}

Inferring relations using logical operators Table 2

\begin{tabular}{|c|c|c|}
\hline Statement & Existing relations & Inferred relations \\
\hline \multirow[t]{4}{*}{$\mathrm{A}:=\mathrm{B}$ and $\mathrm{C}$} & - & $\mathrm{A} \rightarrow \mathrm{B}$ and $\mathrm{A} \rightarrow \mathrm{C}$ \\
\hline & $\mathrm{B} \rightarrow \mathrm{D}$ or $\mathrm{C} \rightarrow \mathrm{D}$ & $\mathrm{A} \rightarrow \mathrm{D}$ \\
\hline & D\#B & D\#A \\
\hline & $\mathrm{D \# C}$ & D\#A \\
\hline \multirow[t]{6}{*}{$\mathrm{A}:=\mathrm{B}$ or $\mathrm{C}$} & - & $\mathrm{B} \rightarrow \mathrm{A}$ and $\mathrm{C} \rightarrow \mathrm{A}$ \\
\hline & $\mathrm{D} \rightarrow \mathrm{B}$ or $\mathrm{D} \rightarrow \mathrm{C}$ & $\mathrm{D} \rightarrow \mathrm{A}$ \\
\hline & $\mathrm{B} \rightarrow \mathrm{D}$ and $\mathrm{C} \rightarrow \mathrm{D}$ & $\mathrm{A} \rightarrow \mathrm{D}$ \\
\hline & $\mathrm{D} \# \mathrm{~B}$ and $\mathrm{D} \# \mathrm{C}$ & $\mathrm{D} \# \mathrm{~A}$ \\
\hline & $\mathrm{B} \rightarrow \mathrm{C}$ & $\mathrm{B} \rightarrow \mathrm{A}$ \\
\hline & $\mathrm{C} \rightarrow \mathrm{B}$ & $\mathrm{C} \rightarrow \mathrm{A}$ \\
\hline \multirow[t]{3}{*}{$\mathrm{A}:=\mathrm{B}$ xor $\mathrm{C}$} & $\mathrm{B} \# \mathrm{C}$ & $\mathrm{B} \rightarrow \mathrm{A}$ and $\mathrm{C} \rightarrow \mathrm{A}$ \\
\hline & $\mathrm{B} \rightarrow \mathrm{D}$ and $\mathrm{C} \rightarrow \mathrm{D}$ & $\mathrm{A} \rightarrow \mathrm{D}$ \\
\hline & $\mathrm{D} \rightarrow \mathrm{B}$ and $\mathrm{D} \rightarrow \mathrm{C}$ & A\#D \\
\hline \multirow[t]{4}{*}{$\mathrm{A}:=\mathrm{B}$ nand $\mathrm{C}$} & $\mathrm{D} \rightarrow \mathrm{B}$ and $\mathrm{D} \rightarrow \mathrm{C}$ & A\#D \\
\hline & $\mathrm{D \# B}$ or $\mathrm{D \# C}$ & $\mathrm{D} \rightarrow \mathrm{A}$ \\
\hline & $\mathrm{B} \rightarrow \mathrm{C}$ & A\#B \\
\hline & $\mathrm{C} \rightarrow \mathrm{B}$ & $\mathrm{A \# C}$ \\
\hline \multirow[t]{4}{*}{$\mathrm{A}:=\mathrm{B}$ nor $\mathrm{C}$} & - & $\mathrm{A \# B}$ and $\mathrm{A \# C}$ \\
\hline & $\mathrm{D} \rightarrow \mathrm{B}$ & A\#D \\
\hline & $\mathrm{D} \rightarrow \mathrm{C}$ & A\#D \\
\hline & $\mathrm{B \# D}$ and $\mathrm{CHD}$ & $\mathrm{B} \rightarrow \mathrm{A}$ \\
\hline \multirow[t]{3}{*}{$\mathrm{A}:=\operatorname{not} \mathrm{B}$} & - & A\#B \\
\hline & $\mathrm{D} \rightarrow \mathrm{B}$ & A\#D \\
\hline & $\mathrm{B} \# \mathrm{D}$ & $\mathrm{D} \rightarrow \mathrm{A}$ \\
\hline- & $\mathrm{A} \rightarrow \mathrm{B}$ and $\mathrm{B} \rightarrow \mathrm{C}$ & $\mathrm{A} \rightarrow \mathrm{C}$ \\
\hline
\end{tabular}

literals for which one of the relations $=, /=<,<=$ $>,>=$ holds. Second, the VHDL logical operators are analyzed (Table 2 ) to infer additional relations between other pairs of control variables. In the table, $A, B, C$, and $D$ are Boolean variables. There are similar rules for pairs of variable assignment statements and for pairs of relations.

\subsection{Orthogonal statements}

All the sequential statements in OBBM may be considered as an if-then-statement. If a statement $Q$ is not conditional one, it may be replaced with if true then $Q$ end if;. Two if-then-statements if VI then $Q 1$ end if; and if $V 2$ then $Q 2$ end if; are defined to be orthogonal if the variables $V 1$ and $V 2$ are orthogonal. One orthogonal statement cannot precede another orthogonal statement. The orthogonal statement bodies are mutually exclusive and may execute on the same functional unit concurrently in the same control step. VHDLlike fragment (4.20) uses seven Boolean variables $V 1, \ldots, V 7$. The relations on the set of variables are described by the matrix:

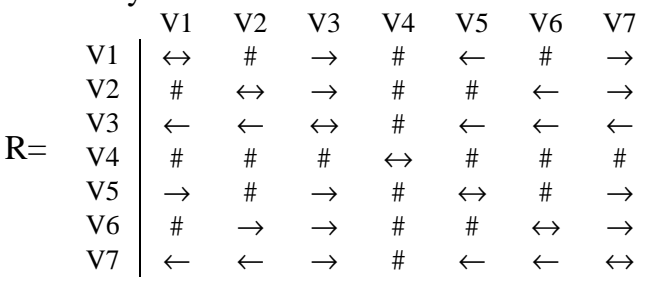

The orthogonal variables graph is presented in Fig.9. The following pairs of control variables are orthogonal: (V3,V4), (V4,V5), (V4,V6), (V5,V6). As a result the pairs of statement sequences as follows are mutually exclusive: $(B, S 1),(S 1, S 2),(S 1, S 3)$, $(S 2, S 3)$.

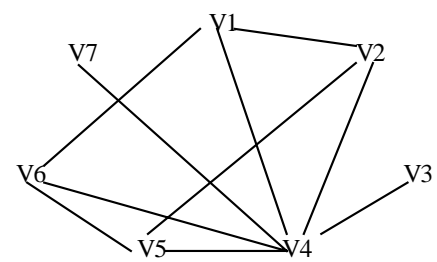

Figure 9. Orthogonal variables graph

\subsection{Operators compatibility and proximity}

There are two cases for operators to be compatible within one control step: the operators belong to orthogonal statements and may execute on the same type of functional unit and the operators are relational and have identical operands. The compatible operators may execute within one control step without additional resources. The operator proximity is used to select compatible operators to be merged. We estimate the proximity of two operators as the number of common inputs and outputs. Maximizing the proximity of operators leads to minimizing the cost of interconnect units in resulting RTL-structure.

\subsection{Precedence of statements}

The statement precedence relation $P R E=V L \cup U S \cup$ $W T$ is a union of three sub-relations:

- A statement $i$ precedes a statement $j((i, j) \in V L)$ if $i$ and $j$ are not orthogonal and $i$ has an output variable that is an input variable for $j$

- A statement $i$ precedes a statement $j((i, j) \in U S)$ if $i$ and $j$ are not orthogonal and $i$ uses a value of an input variable to be assigned a new value by $j$ 
- A statement $i$ precedes a statement $j((i, j) \in W T)$ if a wait-statement $w$ exists such that the pairs $(i, w)$ and $(w, j)$ of statements are not orthogonal and $i$ precedes $w$ and $j$ succeeds $w$ in the VHDL-text.

The relation PRE can be represented as a statement precedence graph. Statements $i$ and $j$ are sequential if a path exists between $i$ and $j$ on the graph, otherwise, the statements are concurrent. The statement precedence graph $P R E$ and its subgraphs $V L, U S$, and $W T$ for the GCD including 12 statements (Fig.7) are shown in Fig.10.

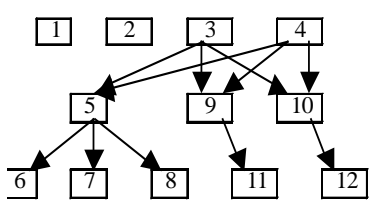

WT

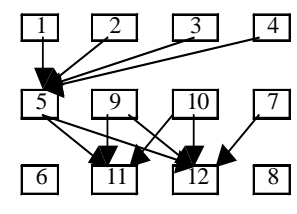

PRE
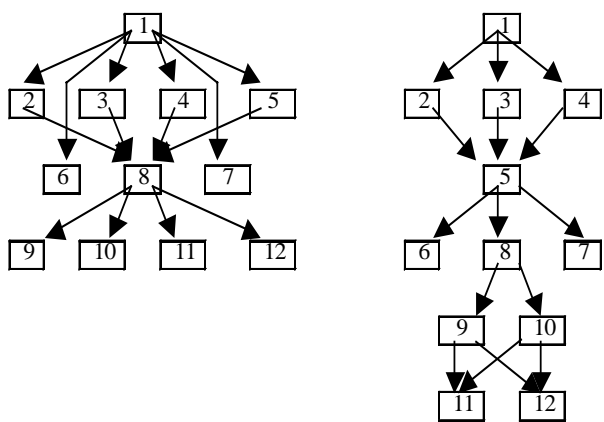

Figure 10. Statement precedence graphs for GCD

\subsection{Extending scheduling techniques}

All the traditional techniques [1-11] scheduling a basic block assume that the sequential statements may not be an if-then-statement and, therefore, may not be orthogonal. Our method allows the orthogonal statements to execute in the same control step in parallel without additional resources. This implies increase in the average number of statements in a step and decrease in the total number of steps. As a result, the number of values computed in one control step and used in another step is reduced as well as the number of registers. The extended ASAP and ALAP being feasible-constrained techniques use the statement precedence graph at input. The modified list scheduling being a resource-constrained technique uses a status of each statement:

- All the predecessors have been already scheduled

- There is a predecessor has not been scheduled

- The statement may be scheduled on an existing functional unit using the orthogonal relation

- Addition of a functional unit is required and others. The modified time-constrained scheduling problem ILPF can be formulated as minimizing $\sum_{k=1}^{m}\left(s_{k} * M_{k}\right)$

subject to

$\sum_{r=1}^{c} z_{r, j, k} \leq M_{k}$

for $1 \leq j \leq s, 1 \leq k \leq m$

$z_{r, j, k}=\min \left(1, \quad \sum y_{r, i, j}\right)$

for $l \leq r \leq c, l \leq j \leq s, 1 \leq k \leq m$

$y_{r, i, j} \leq C_{i, r} * x_{i, j}$

for $1 \leq r \leq c, 1 \leq i \leq n, 1 \leq j \leq s$

$\sum_{r=1}^{c} y_{r, i, j}=1$

for $1 \leq i \leq n, l \leq j \leq s$

$\sum_{j=S_{i}}^{L_{i}} x_{i, j}=1$

for $1 \leq i \leq n$

$\sum_{j=S_{i}}^{L_{i}}\left(j * x_{i, j}\right)-\sum \underset{j=S_{k}}{\left(j * x_{k, j}\right)=1 \quad \text { for all } i \rightarrow k}$

where $n$ is the number of statements; $s$ is the number of control steps; $M_{k}$ and $s_{k}$ are the number and cost of functional units of type $k ; m$ is the number of functional unit types; $C$ is the set of cliques of the orthogonal statements graph; $c$ is the number of cliques in $C$; $C_{i, r}$ equals $l$ if statement $i$ belongs to clique $r ; z_{r, j, k}$ is an integer variable that equals $l$ if at least one statement of clique $r$ is associated with the type $k$ of functional unit and executes in step $j$, otherwise, equals $0 ; y_{r, i, j}$ is a variable that equals 1 if statement $i$ executes in step $j$ and is associated with clique $r$, otherwise, equals $0 ; x_{i, j}$ is a variable that equals $l$ if statement $i$ executes in step $j$, otherwise, equals $0 ; S_{i}$ and $L_{i}$ are the earliest and latest possible time of statement $i ; i \rightarrow k$ denotes $i$ precedes $k$.

\subsection{High-level state machine}

The HLSM initially includes a sequence of states and a set of control variables (Fig.11). Each state has exactly one transition. To speed up the HLSM operation, additional direct transitions are added. Thus, the HLSM for GCD (Fig.12) initially included two sequential states $s O$ and $s l$. All the statements in state $s O$ where covered by an if-thenstatement with the condition $C O$. There was a sense

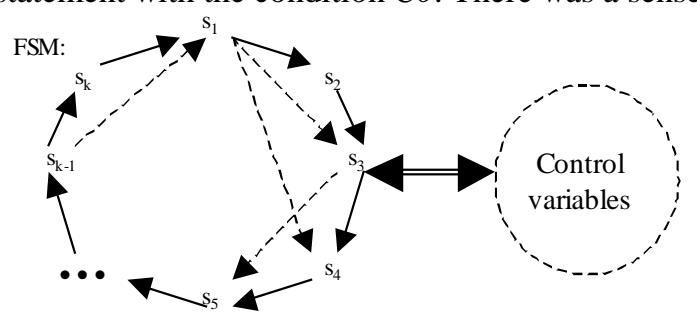

Figure 11. Control part of RTL-structure 
architecture HLSM of GCD is type State_Type is (s0,s1); signal State: State_Type; begin process (Clock,Reset) variable $\mathrm{X}, \mathrm{Y}$ : Bit_Vector(15 downto 0$)$;

variable $C 0, C 2, C 3$ : Boolean:=true; begin

if Reset='0' then State $<=\mathrm{s} 0$; elsif Clock'Event and Clock='1' then case State is

when $\mathrm{s} 0 \Rightarrow$ Ready $<={ }^{\prime} 0$ ' X:=XP; Y:=YP; State $<=\mathrm{s} 1$;

when $\mathrm{s} 1 \Rightarrow \mathrm{C} 0:=\mathrm{X}=\mathrm{Y}$; if $\mathrm{C} 0$ then Ready $<=1$ '; end if;

if $\mathrm{C} 0$ then $\mathrm{Res}<=\mathrm{X}$; end if; $\mathrm{C} 2:=\mathrm{X}<\mathrm{Y} ; \mathrm{C} 3:=\mathrm{X}>\mathrm{Y}$;

if $\mathrm{C} 2$ then $\mathrm{Y}:=\mathrm{Y}-\mathrm{X}$; end if; if $\mathrm{C} 3$ then $\mathrm{X}:=\mathrm{X}-\mathrm{Y}$; end if;

if $\mathrm{C} 0$ then State $<=\mathrm{s} 0$; else State $<=\mathrm{s} 1$; end if;

end case; end if; end process; end HLSM;

Figure 12. High-level state machine for GCD

to come to state $s O$ if and only if $\mathrm{CO}$ equals true. We have added a direct transition from $s 1$ to $s 1$ and replaced the statement $N e x t$ State $<=s O$; in the end of case-statement with the if-statement as shown in Fig. 12 .

\section{Allocation and binding for OBBM}

The RTL-structure being an output of high-level synthesis consists of two parts: a data path (DP) and a finite state machine (FSM). Allocation aims at minimizing the DP cost and defining the set of functional, storage, and interconnect units in the RTL-structure. Binding aims at mapping the elements of behavioral description to the structure components. A lot of allocation and binding techniques have been developed [1-7]: path-based, rule-based, branch and bound, clique partitioning, integer linear programming, simulated-annealing, graph coloring, and other algorithms. The OBBM being a purely data flow representation supports an efficient allocation and binding:

- Analysis of variable lifetime on one basic block

- Generating the variables compatibility relation

- Generating the operators compatibility and proximity relations

- Deriving and folding the DP from the DFG.

\subsection{Variable lifetime analysis}

Assuming that all the statements are distributed on the sequence of HLSM states, a variable $v$ lifetime is represented as an interval $l_{v}=\left[s^{b}{ }_{v}, s^{e}{ }_{v}\right]$ where $s^{b}{ }_{v}$, and $s_{v}{ }_{v}$ are the earliest and latest states in which the variable is alive. In order to compute the interval we use the function Inc: $V \times S \rightarrow\{\varnothing,\{$ in $\},\{$ out $\}$, \{in,out\}\} mapping the pairs variable/state to subsets of the set $\{$ in,out $\}$. It is easy to derive from the function the first state $s_{v}{ }^{\text {first }}$ in which $v$ is used as output and the last state $s_{v}{ }^{\text {last }}$ in which $v$ is used as input. The values $s^{b}{ }_{v}$, and $s_{v}{ }_{v}$ are computed from the values $s_{v}{ }^{\text {first }}$ and $s_{v}{ }^{\text {last }}$ taking into account the fact that the basic block is a body of an infinite loop. The function Inc for the GCD represented as the HLSM in Fig.12 is described in Table 3. The variable lifetimes are $l_{X}=l_{Y}=[s 0, s 1]$ and $l_{C 0}=l_{C 2}=l_{C 3}=[s 1, s 1]$.
Function Inc for GCD lifetime analysis Table 3

\begin{tabular}{|c|c|c|c|c|c|}
\hline \multirow{2}{*}{ State } & \multicolumn{5}{|c|}{ Variable } \\
\cline { 2 - 6 } & $\mathrm{X}$ & $\mathrm{Y}$ & $\mathrm{C} 0$ & $\mathrm{C} 2$ & $\mathrm{C} 3$ \\
\hline S0 & $\begin{array}{c}\text { out }\} \\
\text { \{out }\}\end{array}$ & $\varnothing$ & $\varnothing$ & $\varnothing$ \\
\hline \multirow{2}{*}{ S1 } & $\begin{array}{c}\{\text { in, } \\
\text { out }\}\end{array}$ & $\begin{array}{c}\text { \{in, } \\
\text { out }\}\end{array}$ & $\begin{array}{c}\{\text { in, } \\
\text { out }\}\end{array}$ & $\begin{array}{c}\text { in, } \\
\text { out }\}\end{array}$ & $\begin{array}{c}\{\text { in, } \\
\text { out }\}\end{array}$ \\
\hline
\end{tabular}

6.2. Variable and operator compatibility

A variable $v \in V_{W}$ which is alive within one HLSM state is implemented as a wire. A variable $v \in V_{M}$ which is alive in several HLSM states is mapped to a register, RAM, or ROM. Two variables $v_{l}$ and $v_{2}$ of $V_{M}$ may be mapped to the same storage unit if they are compatible. The variables are compatible if either their lifetime intervals are not intersected or each statement using $v_{l}$ is orthogonal to each statement using $v_{2}$. We describe the variable compatibility as a binary relation $C_{V}$. The operator compatibility is represented by a binary relation $C_{O}$ that is computed using the formula:

$C_{O}=\left(U_{O} \cap \sim\left(S_{O} \backslash O_{O}\right)\right) \cup R_{O}$,

where ' $Y$ ' is a set subtraction operation; $\sim A$ is complementation of set $A ; U_{O}$ is the set of pairs of operators executed on the same type of functional unit; $S_{O}$ is the set of pairs of operators executed in the same HLSM state; $O_{O}$ is the orthogonal relation; $R_{O}$ is the set of pairs of relational operators which execute in the same HLSM state and use the same operands. The operator proximity is estimated as it was described in section 5.4 and represented with a relation $P_{O}$. Five operators are used in the GCD HLSM shown in Fig.11: \{'=', '<', '> ', '-', '-'\}. The relations $C_{O}, U_{O}, S_{O}, O_{O}$, and $R_{O}$ on the set are presented in Fig.13.

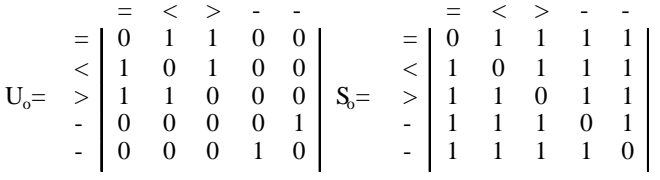

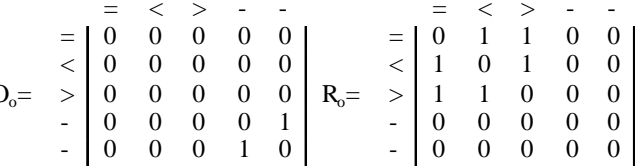

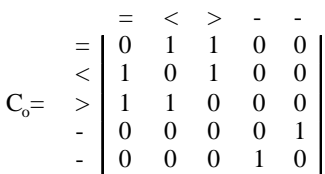

Figure 13. Compatibility relations for GCD

\subsection{Folding data flow graph (data path)}

Folding the DFG and DP aims at minimizing the design cost. The folding scheme is shown in Fig.14. Two types of optimization algorithms have been developed:

- Global optimization that merges the variables, 


\section{FOLDING}

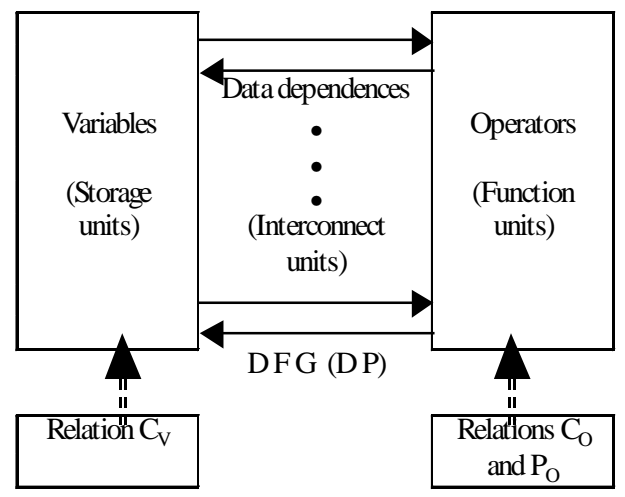

Figure 14: Folding DFG and DP

operators, and data dependencies in parallel

- Local optimization that separately merges the variables, operators, and data dependencies.

The algorithms of the first type find a cheaper design, while of the second type are faster.

\section{Results}

A software has been developed that implements the high-level synthesis techniques based on transformation of VHDL-models. Experimental results have been obtained on several benchmarks. Some advantages and drawbacks of the proposed method are listed in Table 4. Table 5 presents experimental results for three VHDLmodels of the Bubble benchmark [5]. In model 1 all the expressions and conditional statements are split and the iteration scheme of loops is removed. In model 2 the statements located between and after loops are inserted into the loops. In model 3 the loop- and exit-statements are eliminated. The number of control steps is decreased twice due to the transformations. A non-significant increase in the register cost is due to scheduling for minimum register count has not been used. Table 6 proves that the proposed transformations lead to additional possibilities of operations execution in parallel. The use of 2 adders for the original VHDL-model of the Pid benchmark [5] has not decreased the number of control steps. The use of 2 adders for a transformed model has decreased the number of steps from 23 to 16 .

Advantages and drawbacks of OBBM Table 4

\begin{tabular}{|l|l|}
\hline \multicolumn{1}{|c|}{ Advantages } & \multicolumn{1}{|c|}{ Drawbacks } \\
\hline $\begin{array}{l}\text { Reduction in the number of: } \\
\text { - Basic blocks in CFG }\end{array}$ & $\begin{array}{l}\text { The transformed } \\
\text { behavioral VHDL- } \\
\text { - Control steps and FSM states } \\
\text { model differs from } \\
\text { - State transitions }\end{array}$ \\
$\begin{array}{l}\text { - Storage units } \\
\text { - Functional units } \\
\text { specification VHDL- }\end{array}$ \\
$\begin{array}{l}\text { More opportunities for pipelining, } \\
\text { chaining, multi-cycling, and } \\
\text { asynchronous high-level synthesis }\end{array}$ & $\begin{array}{l}\text { Extending the set of } \\
\text { control variables }\end{array}$ \\
\hline
\end{tabular}

Bubble benchmark

Table 5

\begin{tabular}{|l|c|c|c|}
\hline \multirow{2}{*}{ Parameter } & \multicolumn{3}{c|}{ Behavioral VHDL-model } \\
\cline { 2 - 4 } & Bub_1 & Bub_2 & Bub_3 \\
\hline Loops & 9 & 9 & 0 \\
\hline Control steps & 20 & 15 & 10 \\
\hline Registers & 7 & 9 & 10 \\
\hline Register width (bits) & 104 & 106 & 107 \\
\hline Multiplexers & 4 & 4 & 6 \\
\hline Multiplexer width (bits) & 68 & 68 & 70 \\
\hline Multiplexer inputs & 13 & 14 & 18 \\
\hline RAM & 1 & 1 & 1 \\
\hline
\end{tabular}

Pid benchmark Table 6

\begin{tabular}{|l|c|c|c|}
\hline \multirow{2}{*}{\multicolumn{1}{c|}{ Parameter }} & Model 1 & \multicolumn{2}{c|}{ Model 2 } \\
\cline { 2 - 4 } & $1-2$ adds & 1 adder & 2 adds \\
\hline Control steps & 23 & 22 & 16 \\
\hline Registers & 13 & 16 & 15 \\
\hline Register width (bits) & 389 & 423 & 422 \\
\hline Multiplexers & 8 & 8 & 10 \\
\hline Multiplexer width (bits) & 227 & 227 & 291 \\
\hline Multiplexer inputs & 33 & 34 & 40 \\
\hline Collectors & 9 & 9 & 9 \\
\hline ROM & 1 & 1 & 1 \\
\hline
\end{tabular}

\section{References}

[1] R.A.Bergamaschi, "High-Level Synthesis in a Production Environment", Fundamentals and Standards in Hardware Description Languages, J.P. Mermet, ed., Kluwer Academic Publishers, Norwell, Mass., 1993, pp.195-230.

[2] R.Camposano and W.Rosensteil, "Synthesizing Circuits from Behavioral Descriptions", IEEE Trans. CAD, Vol.CAD-8, Feb. 1989, pp.171-180.

[3] D.D.Gajski et al., "High-Level Synthesis: Introduction to Chip and System Design", Kluwer Academic Publishers, Norwell, Mass., 1992.

[4] T.Hwang, J.Lee, Y.Hsu, "A Formal Approach to the Scheduling Problem in High- Level Synthesis", IEEE Trans.on CAD, Vol.10, No.4, 1991.

[5] A.A.Jerraya, I.Park, and K.O'Brien, "Amical: An Interactive High-Level Synthesis Environment", Proc. European Design Automation Conf.93, IEEE Computer Society Press, Los Alamitos, Calif., 1993.

[6] A.Prihozhy, "Net Scheduling in High-Level Synthesis", IEEE Design \& Test of Computers, Spring, 1996, pp.26-35.

[7] A.Prihozhy "Asynchronous Scheduling and Allocation”, Proc. DATE 98, IEEE CS Press, CA, 1998.

[8] A.Prihozhy and F.Buijs "Transformations of Behavioral VHDL-Descriptions", National Academy of Sciences, Belarus, 1994.

[9] A.Prihozhy, "Methods for Logical Algorithm Equivalent Transformation in VLSI CAD", Trans. Physics \& Mathematics, National Academy Sciences, Belarus, 1992, N 2, pp.86-92. (in Russian).

[10] W.Rosensteil, "Experiences with High-Level Synthesis from VHDL-Specifications", Proc. Workshop on Design Methodologies for Microelectronics and Signal Processing, GliwiceCracow, 1993, pp.405-412.

[11] E.Villar and P.Sanches, "Synthesis Applications of VHDL", Fundamentals and Standards in Hardware Description Languages, J.P. Mermet, ed., Kluwer Academic Publishers, 1993, pp.231-262. 